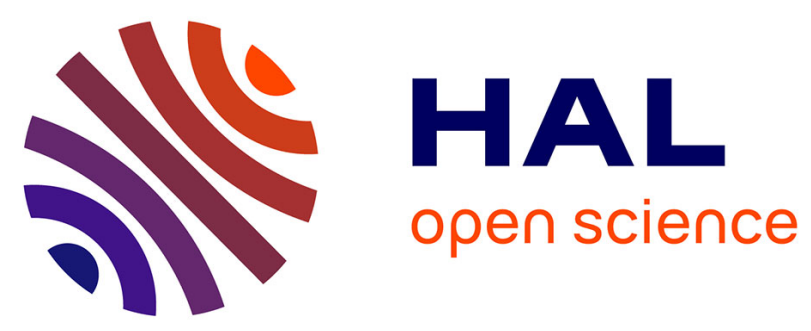

\title{
High spectral purity laser characterization with a self-heterodyne frequency discriminator
}

Olivier Llopis, Zeina Abdallah, Vincent Auroux, Arnaud Fernandez

\section{To cite this version:}

Olivier Llopis, Zeina Abdallah, Vincent Auroux, Arnaud Fernandez. High spectral purity laser characterization with a self-heterodyne frequency discriminator. 2015 Joint conference of the IEEE International Frequency Control Symposium and European Frequency and Time Forum, Apr 2015, Denver, United States. hal-01141233

\section{HAL Id: hal-01141233 https://hal.science/hal-01141233}

Submitted on 10 Apr 2015

HAL is a multi-disciplinary open access archive for the deposit and dissemination of scientific research documents, whether they are published or not. The documents may come from teaching and research institutions in France or abroad, or from public or private research centers.
L'archive ouverte pluridisciplinaire HAL, est destinée au dépôt et à la diffusion de documents scientifiques de niveau recherche, publiés ou non, émanant des établissements d'enseignement et de recherche français ou étrangers, des laboratoires publics ou privés. 


\title{
High spectral purity laser characterization with a self-heterodyne frequency discriminator
}

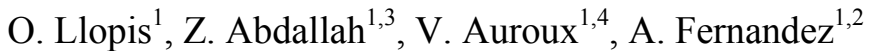 \\ ${ }^{1}$ CNRS, LAAS, Univ. de Toulouse, Toulouse, France \\ ${ }^{2}$ Univ. de Toulouse, UPS, Toulouse, France \\ ${ }^{3} \mathrm{CNES}$, Toulouse, France ; ${ }^{4}$ OSAT, Toulouse, France \\ E-mail: llopis@laas.fr
}

\begin{abstract}
The performance of a delay line discriminator dedicated to the characterization of high spectral purity lasers is investigated. The system noise floor is obtained using a symmetrical delay. The vibrations are one of the main problems with this approach at the low frequency offsets. However, the system is able to characterize commercially available narrow linewidth lasers, such as external cavity lasers and fiber lasers.
\end{abstract}

Keywords-laser; frequency noise; phase noise; metrology; frequency discriminator

\section{INTRODUCTION}

The today availability of small size narrow linewidth lasers, such as semiconductor lasers optically coupled to high quality factor optical resonators (WGM resonators, fiber resonators...), is an opportunity to develop new embedded applications for which the laser phase noise level is mandatory. This is the case, as an example, of high precision interferometers and of microwave to terahertz signal generation using optics. Before being used in these systems, this type of laser has to be characterized in terms of phase or frequency noise. However, phase noise measurement in the optical domain is not as developed as it is for radio frequency (RF) sources, although some commercial systems are already available (ex : OEwaves). The reason is that it is much more difficult to design a high quality optical frequency synthesizer than a low phase noise RF synthesizer. Therefore, the easier way to measure this type of sources is still to set up the frequency discriminator technique.

In this approach, the laser signal is split in two paths, one path is delayed with a delay smaller than the coherence length of the laser (contrarily to linewidth measurement case) and the signals are combined on a photodiode [1-4]. In the selfheterodyne case, an acousto-optic modulator is added in one of the paths in order to shift the signal frequency around a few tens of MHz. In this case, the noise is analyzed using an RF phase noise test bench at this frequency [3-5], which prevents the detection of some parasitic contributions of low frequency noise, such as the laser 1/f amplitude noise (but also increase the system cost and complexity).

The main difficulty in this approach is to evaluate the measurement noise floor. In microwave frequency discriminators, it is measured using a bypass of the delay line. However, in the optical case, part of the noise may come from nonlinear effects in the fiber [6], or from vibration sensitivity of the fiber spool $[7,8]$ or of the whole system, and the noise floor has to be measured with the fiber maintained in the system.

In this paper, measurements of two types of high spectral purity lasers using a self-heterodyne system are presented. Then, the noise floor of the system is evaluated using a set of two identical fiber spools. Finally, we focus on the choice of the delay and on the importance of the reduction of vibration sensitivity.

\section{MEASUREMENT SYSTEM}

The measurement system is depicted in Figures 1 and 3 . The laser signal is split in two paths. On one path, an acoustooptic modulator shifts the laser frequency of $80 \mathrm{MHz}$. On the other path, the signal is delayed using a fiber spool, and then recombined to the first path. A polarization controller is added after the fiber spool in order to improve the output signal level. The beat frequency is recovered using a photodiode, amplified and, finally, feeds an RF phase noise measurement bench (in our case, an Agilent E5052B).

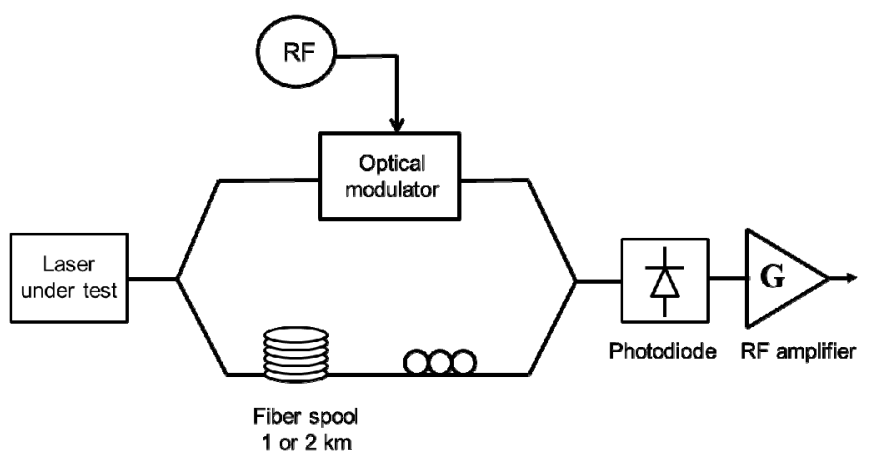

Fig 1 : Self heterodyne frequency noise measurement system

Using this approach, the measured RF phase noise at $80 \mathrm{MHz}$ is proportional to the laser frequency noise, at least at offset frequencies close to the carrier $[3,4]$. Far from the 
carrier, the output spectrum is attenuated with a classical $\sin \mathrm{x} / \mathrm{x}$ shape. This is clearly visible in Figure 2, in which the phase noise of the $80 \mathrm{MHz}$ output is depicted in case of an external cavity semiconductor laser (RIO Inc.) feeding the bench, and using a $2 \mathrm{~km}$ fiber spool in the bench. At $100 \mathrm{kHz}$, the measurement sensitivity locally drops down to zero because of the first zero of the $\sin \mathrm{x} / \mathrm{x}$ function.

Because of this response, we have limited our measurements on the $1 \mathrm{~Hz}-100 \mathrm{kHz}$ offset range, and we have corrected the measured data using the reverse function of the $\sin x / x$ response of the bench and the time delay of the spools we are using. More precisely, the laser frequency noise can be computed from the measured $80 \mathrm{MHz}$ RF phase noise [3] using equation 1.

$$
\mathrm{S}_{\Delta \mathrm{f} \text { in } \mathrm{Hz} / \sqrt{\mathrm{Hz}}}=\frac{\mathrm{f}_{\mathrm{m}}}{\sqrt{2} \sin \left(\pi \mathrm{f}_{\mathrm{m}} \tau\right)} 10^{\mathrm{L}_{\mathrm{RF}}\left(\mathrm{f}_{\mathrm{m}}\right) / 20}
$$

were $f_{m}$ is the offset frequency, $\tau$ is the time delay between the two paths and $\mathrm{L}_{\mathrm{RF}}\left(\mathrm{f}_{\mathrm{m}}\right)$ is the measured single sideband phase noise, in $\mathrm{dB}_{\mathrm{c}} / \mathrm{Hz}$, of the RF signal.

Another important result of equation 1 is that it gives the proportionality coefficient between the RF phase noise and the laser close to carrier frequency noise, and that this coefficient is itself proportional to the delay $\tau$. Thus, even if a large delay limits the measurement bandwidth because of the $\sin \mathrm{x} / \mathrm{x}$ effect, it enhances the measurement sensitivity and, as we will see, reduces the noise floor.

Equation 1 is used to compute the laser frequency noise data. However, when a noise floor is measured instead of a true laser frequency fluctuation, this noise floor is not affected by the $\sin \mathrm{x} / \mathrm{x}$ response, because it is either a noise superimposed on the system output, or the demodulation of the laser amplitude noise. Therefore, to convert the measured noise floor into frequency noise, we use a constant coefficient which is the limit of the above coefficient at low frequencies.

$$
\mathrm{S}_{\Delta \mathrm{f} \text { noise floor }}=\frac{1}{\sqrt{2} \pi \tau} 10^{\mathrm{L}_{\mathrm{RF}}\left(\mathrm{f}_{\mathrm{m}}\right) / 20}
$$

This frequency discriminator has been used to measure two different high spectral purity lasers: a semiconductor laser stabilized on an external resonator, available in a small butterfly package (RIO), and a laboratory high quality fiber laser (Koheras). Both lasers are used at a relatively low output power: $7 \mathrm{~mW}(60 \mathrm{~mA})$ for the RIO laser and $16 \mathrm{~mW}$ for the Koheras laser. The power dependence of the spectral purity is relatively weak for this type of lasers, and such an output power is largely sufficient to drive our bench (however, a small improvement of about 2 to $3 \mathrm{~dB}$ is observed on the RIO laser phase noise when biased at $120 \mathrm{~mA}$ instead of $60 \mathrm{~mA}$ ).

Figure 4 depicts the result of the measurements performed on these two lasers, under these conditions. The measurement is performed in a Faraday's shielded room using the E5052 system and the frequency noise is computed using equation 1 .

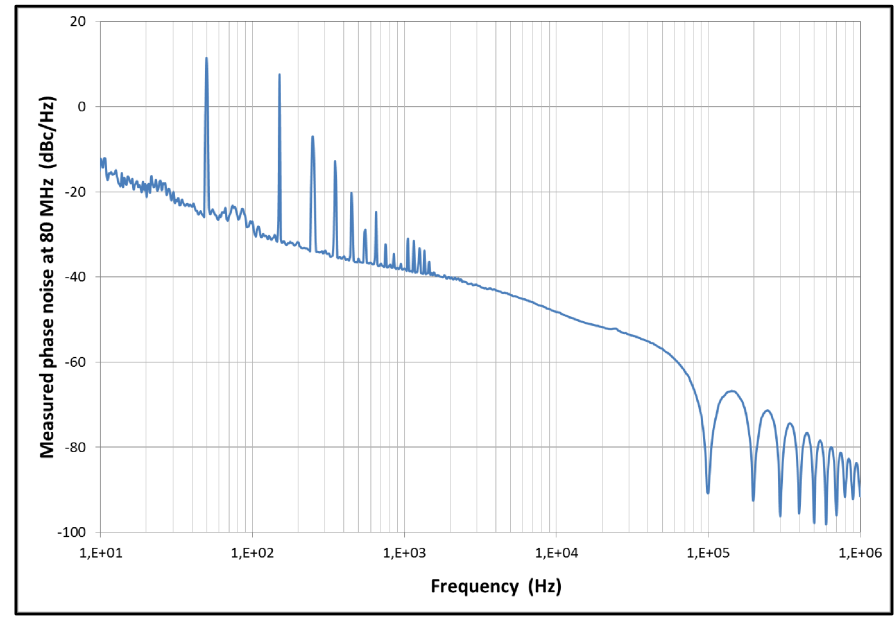

Fig 2 : RF phase noise data at $80 \mathrm{MHz}$ measured for a semiconductor laser stabilised on an external resonator with a $2 \mathrm{~km}$ discriminator

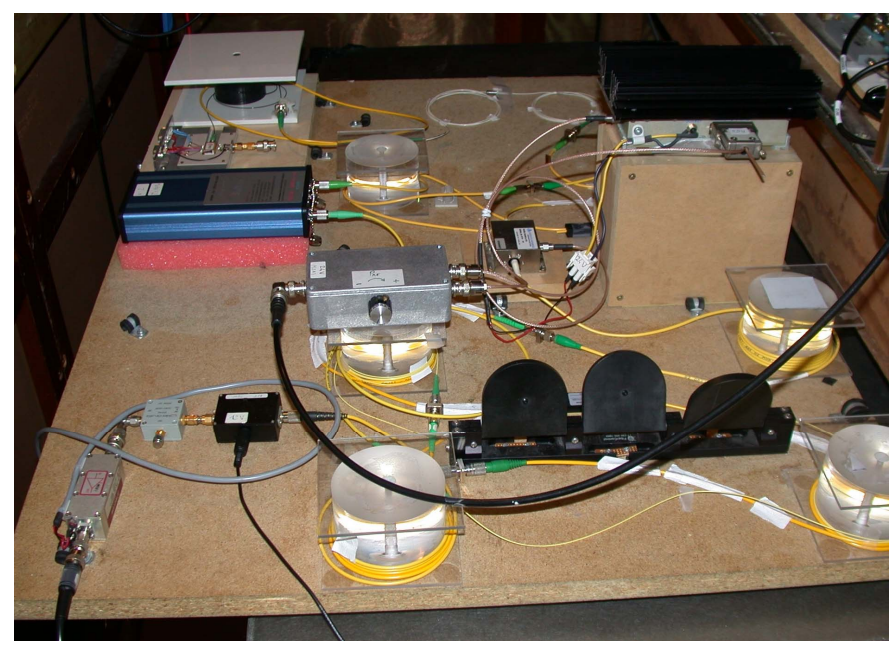

Fig 3 : Measurement bench with the $2 \mathrm{~km}$ spool (blue package, on the left)

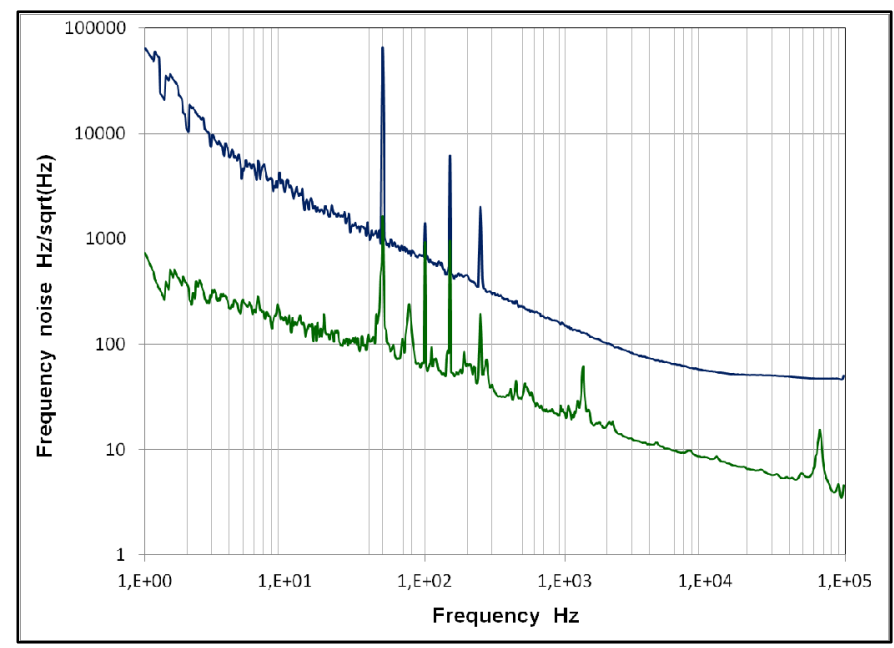

Fig 4 : Measured frequency noise of two high spectral purity lasers : external cavity miniature laser (above) and laboratory fiber laser (below) 
In Figure 5, the same measurements are plotted in terms of single sideband phase noise, which shows the difference in $\mathrm{dB}$ between these two lasers, and also give an idea of the laser spectral shape, taking into account that when the phase noise is sufficiently low compared to $0 \mathrm{~dB}$, it represents the lateral noise wings of the spectrum (we do not want to compute the linewidth, because this parameter is too much dependent on the integration time and has, in our opinion, no rigourous meaning for this type of high spectral purity optical sources).

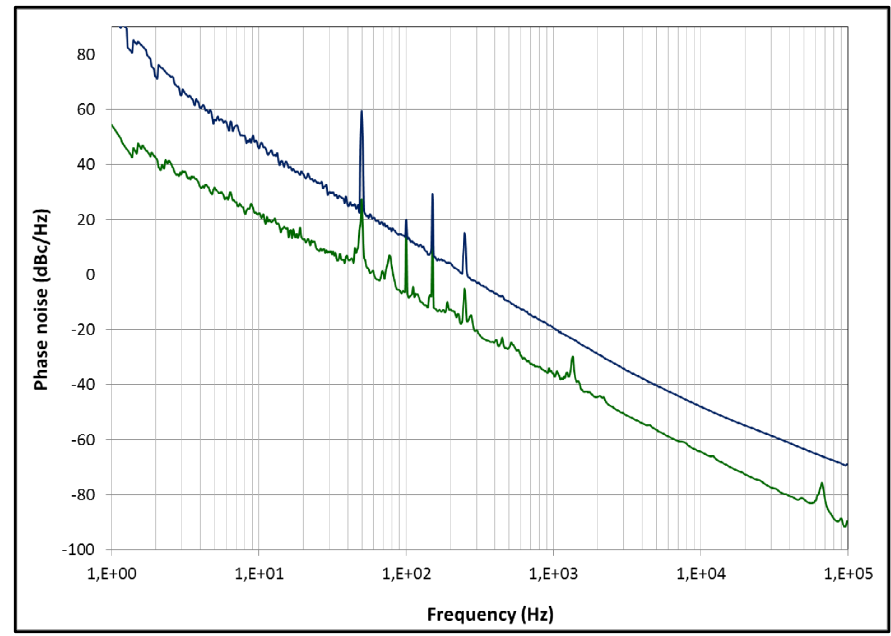

Fig 5 : Phase noise in $\mathrm{dBc} / \mathrm{Hz}$ computed for the two lasers of Fig. 4

In a preceding paper [4], the phase noise of the Koheras laser had been a few $\mathrm{dB}$ overestimated in the $10 \mathrm{~Hz}-100 \mathrm{~Hz}$ range, because of a vibration noise sensitivity of the fiber spool we were using. Indeed, like in any type of noise measurement, it is essential to determine if the measured data are not too close from the measurement bench noise floor. The next paragraph is dedicated to the evaluation and the optimisation of this noise floor.

\section{MEASUREMENT NOISE FLOOR}

An obvious technique to measure a frequency discriminator noise floor is to cancel the delay $\tau$ by removing the fiber spool. In this case, the system sensitivity to the laser frequency noise is null, and only remains the other noise contributions. However, such an evaluation of the noise floor is quite optimistic. Indeed, it also cancels any noise contribution coming from the fiber spool. There are mainly three types of low frequency noise that can be generated in a fiber spool: noise related to optical nonlinear effects [6], such as Stimulated Brillouin Scattering (SBS) ; diffusion noise on impurities, such as Rayleigh scattering noise ; noise due to a vibration sensitivity of the spool $[7,8]$.

Therefore, in order to get a more precise evaluation of the noise floor, two identical fiber spools have been used to cancel the delay $\tau$, one in each path of the interferometer. In this case, the above described effects can still be observed, and are included in the real noise floor.
The plastic rod spool used in our first experiments [4] has been replaced by a packaged spool (Fig. 3). The fiber is free of constraint in this package, but it is however mechanically maintained thanks to the relative small size of the package and to an inside plastic protection. Two set of spools have been used, one of $1 \mathrm{~km}(\tau=5.1 \mu \mathrm{s})$ and one of $2 \mathrm{~km}(\tau=10.05 \mu \mathrm{s})$.

Figure 6 depicts the results of the noise floor measurements in the $2 \mathrm{~km}$ fiber spool case, with the battery biased RIO laser feeding the bench $(7 \mathrm{~mW}$ optical input power). A clear contribution of vibration noise is observed between $10 \mathrm{~Hz}$ and $2 \mathrm{kHz}$ when the system is set up on a normal table (green curve). When the system is put on a low vibration table (but still inside the Faraday shielded room), most of the vibration contribution is reduced and the noise floor measured with the two spools technique (red curve) is close to the noise floor measured with the spool removed from the bench (two harms with short delay; black curve).

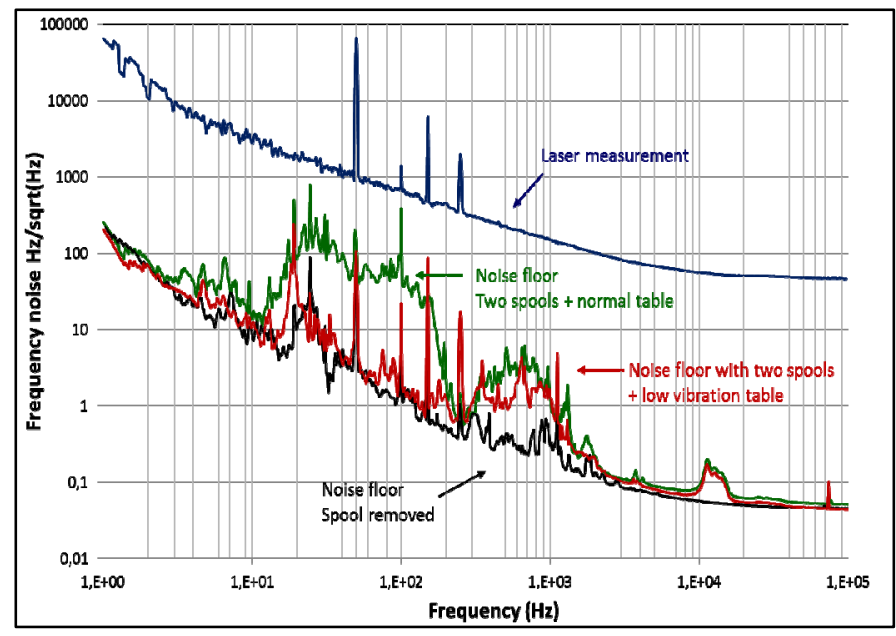

Fig 6 : Noise floor measured for the $2 \mathrm{~km}$ delay bench with the RIO laser, using three different configurations : no spool (black); two spools and normal table to support the bench (green); two spools and low vibration table (red).

These observations allow us to clarify the importance of the vibration noise contribution to the noise floor, and to demonstrate the absence of non-linear optical noise contribution. Indeed, in both cases (Koheras or RIO lasers), we are feeding the fiber spool with optical power levels much below the SBS threshold, which is in the range of $30 \mathrm{~mW}$ for a $2 \mathrm{~km}$ fiber spool [9].

The same experiments have been performed with the $1 \mathrm{~km}$ spools. On Figure 7, it is clear that the vibration problem is still observed with the $1 \mathrm{~km}$ spool. Finally, with this configuration, the noise floor is increased of about $6 \mathrm{~dB}$, which is directly related to the reduction of the delay $\tau$ of a factor 2 , and thus of the same reduction in sensitivity.

Finally, the $2 \mathrm{~km}$ spool appears to be a good compromise between a good sensitivity, a high SBS threshold and a large offset frequency bandwidth, and this fiber length is selected for further experiments.

The last question is: what is limiting the noise floor when the fiber spool is removed. At high frequency offsets, it is 
clear that the signal to noise ratio may have an influence, and this signal to noise ratio is mainly determined by the laser RIN at the IF frequency $(80 \mathrm{MHz})$. However, close to the carrier, we also found an influence of the oscillator which is driving the acousto-optic modulator. This oscillator features a $-43.5 \mathrm{dBc} / \mathrm{Hz}$ phase noise level at $1 \mathrm{~Hz}$, with a $30 \mathrm{~dB} / \mathrm{dec}$ slope in this region. It determines the optical frequency discriminator noise floor between $1 \mathrm{~Hz}$ and $5 \mathrm{~Hz}$ offset, as shown in Figure 8. However, between $5 \mathrm{~Hz}$ and $2 \mathrm{kHz}$, the noise floor seems still to be determined by vibration sensitivity. Finally, between $2 \mathrm{kHz}$ and $100 \mathrm{kHz}$, the noise floor changes with the laser type and power, which means that it should be mostly related to the laser RIN.

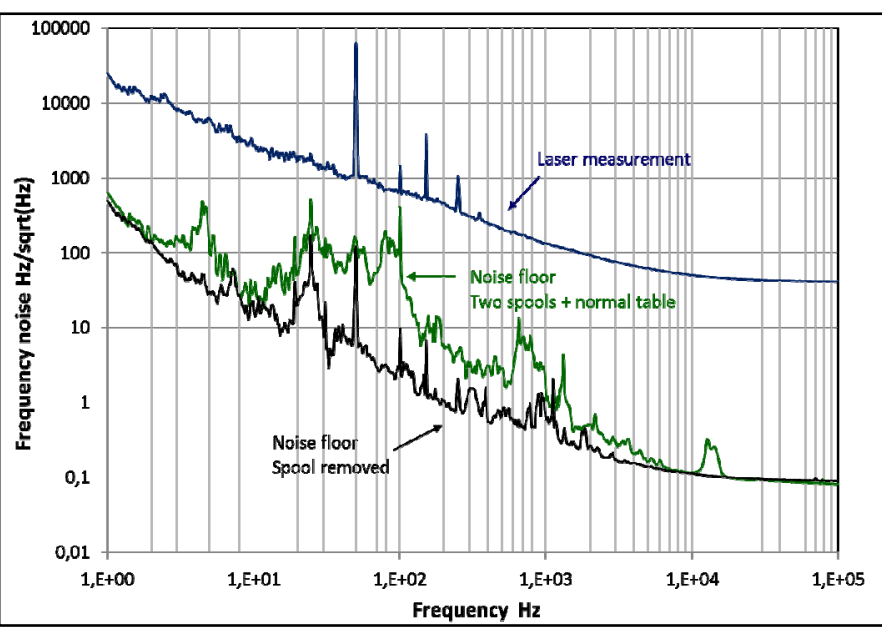

Fig 7 : Noise floor measured for the $1 \mathrm{~km}$ delay system with the RIO laser, using two different configurations : no spool (black) and two spools and normal table to support the experiment (green).

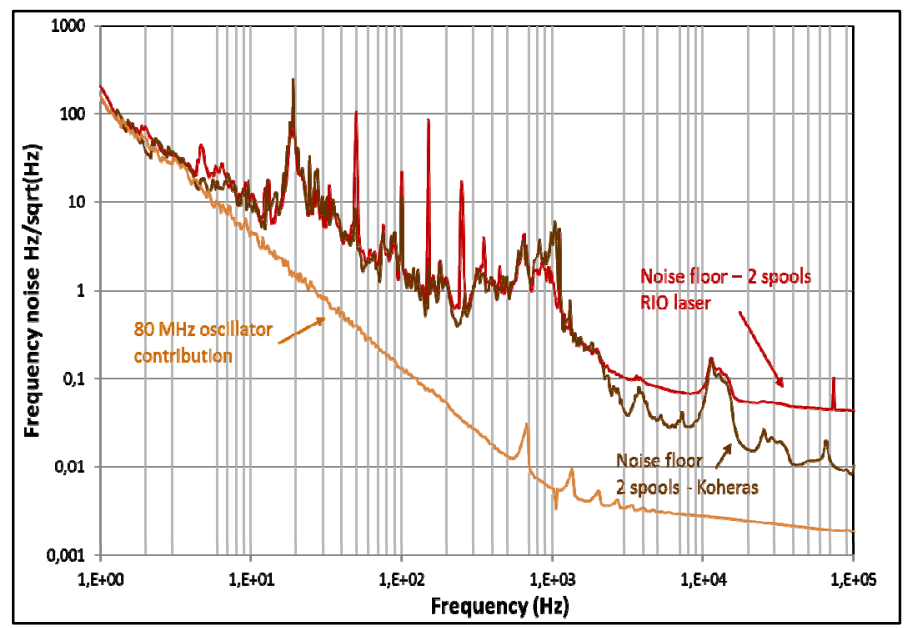

Fig 8 : Noise floor measured with two spools of $2 \mathrm{~km}$ on a low vibration table for two different lasers, and contribution of the $80 \mathrm{MHz}$ oscillator.

The next step is now to improve the experimental set up to overcome these limitations. The problem of the acousto-optic modulator driver is easy to solve: this source will be replaced by a high spectral purity oscillator (a high quality OCXO) followed by a high power amplifier, and this should lead to a $30 \mathrm{~dB}$ improvement of the $1 \mathrm{~Hz}$ phase noise (case of a vibration free experiment). However, the noise between $10 \mathrm{~Hz}$ and $2 \mathrm{kHz}$ will be more difficult to improve: it is directly related to the interferometer sensitivity to vibrations. A new set up will be designed, featuring higher compactness, and on which mechanical isolation will be easier to provide.

\section{CONCLUSION}

The measurement of two different high spectral purity lasers has been performed with a delay line frequency discriminator. The problem of the measurement noise floor has been carefully studied and it has been found that it is mainly limited by the mechanical vibration sensitivity of the fiber spool, and also of the whole interferometer set-up. The system sensitivity and dynamic range is however sufficient to investigate on the two examples of lasers presented in this paper. However, for ultra-high spectral purity lasers characterization, the set-up must still be improved.

\section{REFERENCES}

[1] D. Derickson, Fiber optic Test and Measurement, Prentice Hall 1998.

[2] H. Ludvigsen, M. Tossavainen, M. Kaivola, "Laser linewidth measurements using self-homodyne detection with short delay", Optics Communications, vol 155, Oct. 1998, pp. 180-186.

[3] S. Camatel, V. Ferrero, "Narrow linewidth CW laser phase noise characterization methods for coherent transmission system applications", Journal of Lightwave Tech., vol 26, №17, Sept. 2008, pp. 3048-3055.

[4] O. Llopis, P.H. Merrer, H. Brahimi, K. Saleh, P. Lacroix, "Phase noise measurement of a narrow linewidth $\mathrm{CW}$ laser using delay line approaches", Optics Letters, Vol. 36, N 14, July 2011.

[5] F. Kefelian, H. Jiang, P. Lemonde, G. Santarelli, "Ultralow frequency noise stabilization of a laser by locking to an optical fiber delay line", Optics Letters, Vol 34, N 7, April 2009, pp. 914-916.

[6] O. Okusaga, J. Cahill, W. Zhou, A. Docherty, G.M. Carter, C.R. Menyuk, "Optical scattering induced noise in RF-photonic systems," Proc. of the Int. Freq. Control Symp. and European Freq. and Time Forum (IFCS-EFTF), San Francisco, May 2011.

[7] T. Li, B. Argence, A. Haboucha, H. Jiang, J.L. Dornaux, D. Koné, A. Clairon, P. Lemonde, G. Santarelli, C. Nelson, A. Hati, E. Burt, "Low vibration sensitivity fiber spools for laser stabilization", Proc. of the Int. Freq. Control Symp. and European Freq. and Time Forum (IFCS-EFTF), San Francisco, May 2011.

[8] A. Hati, C.W. Nelson, J. Taylor, N. Ashby, D.A. Howe, "Cancellation of vibration induced phase noise in optical fibers", IEEE Photonics Tech. Letters, Vol 20, N²2, November 2008, pp. 1842-1844.

[9] G.P. Agrawal, Fiber-Optic Communication Systems, Wiley Ed., 1997. 